\title{
The role of spatial and temporal radiation deposition in inertial fusion chambers: the case of HiPER
}

\author{
J. Alvarez, D. Garoz, R. Gonzalez-Arrabal, A. Rivera and \\ M. Perlado
}

Instituto de Fusión Nuclear, UPM, Madrid, Spain

\begin{abstract}
The first wall armour for the reactor chamber of HiPER will have to face short energy pulses of 5 to $20 \mathrm{MJ}$ mostly in the form of $x$-rays and charged particles at a repetition rate of $5-10 \mathrm{~Hz}$. Armour material and chamber dimensions have to be chosen to avoid/minimize damage to the chamber, ensuring the proper functioning of the facility during its planned lifetime. The maximum energy fluence that the armour can withstand without risk of failure, is determined by temporal and spatial deposition of the radiation energy inside the material. In this paper, simulations on the thermal effect of the radiation-armour interaction are carried out with an increasing definition of the temporal and spatial deposition of energy to prove their influence on the final results. These calculations will lead us to present the first values of the thermo-mechanical behaviour of the tungsten armour designed for the HiPER project under a shock ignition target of $48 \mathrm{MJ}$. The results will show that only the crossing of the plasticity limit in the first few micrometres might be a threat after thousands of shots for the survivability of the armour.
\end{abstract}

(Some figures in this article are in colour only in the electronic version)

\section{Introduction}

Fusion reactions in inertial confinement devices are characterized by short (some ns long) and very energetic explosions (from some tens to some hundred of MJ) which yield neutrons, gamma and $\mathrm{x}$-rays and high energy ions. Among those products, neutrons account for around $70 \%$ of the fusion energy and show almost no interaction with the first wall of the reaction chamber. If no protection scheme is devised (ion deflector or a high $Z$ gas), the remaining $30 \%$ of the energy in the form of $x$-rays and ions is deposited on the inner wall of the chamber and the front-end optics. Thus, drywall designs rely on large chamber dimensions (usually 5-6 m in radius) and an inner wall armour to typically withstand heat loads of $1-6 \mathrm{~J} \mathrm{~cm}^{-2}$ and powers of some $\mathrm{GW} \mathrm{m}^{-2}$ [1]. From the thermo-mechanical point of view, this armour has to be made of a material with a high thermal conductivity and melting/sublimation point and with good properties to mechanical stress and fatigue. The goal is to avoid or at least to minimize the mass loss and cracking leading to mechanical failure. The materials under consideration for that purpose are tungsten and carbon based composites. However, nowadays, the tritium retention problem of carbon compounds makes tungsten the standard option on most armour designs [2]. A look at the bibliography shows that the now cancelled American HAPL project [3] relied on tungsten armour for the
7.5 m radius chamber to absorb the energy from $150 \mathrm{MJ}$ targets (average wall load $5.5 \mathrm{~J} \mathrm{~cm}^{-2}$ ) at a 5-10 $\mathrm{Hz}$ repetition rate. The Japanese Falcon D design [4] also considered tungsten as the most adequate armour material for the reaction chamber. In their design, they planned a 5-6 $\mathrm{m}$ radius chamber to house $40 \mathrm{MJ}$ (an average wall load of $2 \mathrm{~J} \mathrm{~cm}^{-2}$ ) fusion targets at a repetition rate of $30 \mathrm{~Hz}$. The European inertial fusion project, $\mathrm{HiPER}^{1}$, is meant to use targets of intermediate energies (some tens of MJ up to $100 \mathrm{MJ}$ maximum) at a repetition rate of 5 to $10 \mathrm{~Hz}$. For an initially planned chamber of $5 \mathrm{~m}$ radius and a $50 \mathrm{MJ}$ target, tungsten will have to accommodate heat loads of around $4 \mathrm{~J} \mathrm{~cm}^{-2}$ per shot for an estimated lifetime of a few thousand shots.

This paper presents the first simulation numbers for the HiPER project on the thermo-mechanical behaviour of tungsten armour for the proposed $5 \mathrm{~m}$ radius chamber under the explosion of a shock ignition target of $48 \mathrm{MJ}$. First, the characterization of the products of a shock ignition target is described. Then, a series of simulations is presented to highlight the importance of proper modelling of the time profile of the delivered energy and its spatial deposition. Finally, the resulting thermo-mechanical behaviour of the tungsten armour when the spatial and temporal profile of the energy deposition is accurately accounted for is shown. Conclusions based on

1 http://www.hiper-laser.org/ 
Table 1. Energy distribution of a $48 \mathrm{MJ}$ shock ignition target.

\begin{tabular}{llr}
\hline & $\begin{array}{l}\text { Energy (J) } \\
\text { Total }=48 \times 10^{6}\end{array}$ \\
\hline X-rays & \multicolumn{1}{l}{} \\
\hline Neutrons & $6.8 \times 10^{5}$ & $1.42 \%$ \\
Deuterons & $3.6 \times 10^{7}$ & $75.03 \%$ \\
Tritons & $2.9 \times 10^{6}$ & $6.04 \%$ \\
$\mathrm{He}$ & $3.5 \times 10^{6}$ & $7.29 \%$ \\
$\mathrm{C}$ & $3.6 \times 10^{6}$ & $7.5 \%$ \\
Gamma rays $\mathrm{H},{ }^{3} \mathrm{He},{ }^{13} \mathrm{C}$. & $3 \times 10^{6}$ & $2.08 \%$ \\
\hline
\end{tabular}

the simulation results reveal that, except for possible damage due to fatigue, the selected tungsten armour will withstand the thermo-mechanical effects of a $50 \mathrm{MJ}$ fusion target. Blistering due to He retention is eventually the main cause of $\mathrm{W}$ armour failure [5].

\section{Fusion products of a shock ignition target}

Fusion targets are filled with deuterium and tritium which, when compressed and ignited, generate $14.1 \mathrm{MeV}$ neutrons and 3.5 MeV He ions. Through different processes, that energy is redistributed among the non-burnt ions (D and T and atoms from the target plastic cover, typically $\mathrm{C}$ and $\mathrm{H}$ ) and in the form of x-rays. To study the thermo-mechanical effects of the chamber armour against this radiation, the total amount of energy deposited on the wall is not sufficient. As shown later, it is necessary to accurately know the distribution and energy spectra of the different products which can be calculated using a radiation-hydrodynamic code.

One of the goals of the HiPER project is to reduce the required laser energy to achieve fusion compared with current approaches (for example, NIF [6]). Thus, HiPER is initially opting for fast or shock ignition targets which, in principle, require around $1 / 3$ of the energy of the central ignition targets. For the studies presented in this work, we have chosen the product spectra of a $48 \mathrm{MJ}$ shock ignition target obtained using the LASNEX code $[7,8]$. In table 1 , the energy distribution among the different species is summarized and one can already identify the most relevant particles in the plasma-armour interaction. Since neutrons do not interact with the armour, deuterium, tritium and helium are the main species responsible for the delivery of energy on the armour. The contribution of carbon atoms to the total deposition of energy on the wall will be obviated in the thermo-mechanical study. (Carbon content and its energy spectrum is very dependent on the target design and its inclusion would influence the validity of the results for a general case). X-rays deliver only $1.4 \%$ of the fusion energy to the armour but, as will be shown, they play an important role in the simulations due to their prompt deposition of energy and consequently high power load on the walls. Other species such as gamma rays, protons and isotopes will be excluded for their minor effect.

Thus, figure 1 shows a detailed description of the distribution and energy spectra of the relevant particles to our thermo-mechanical study of the armour. D,T, He and x-rays deliver in total an energy of $10.7 \mathrm{MJ}$ to the wall.

As it will be shown later, the characterization of the temporal profile of energy deposition is required for a proper
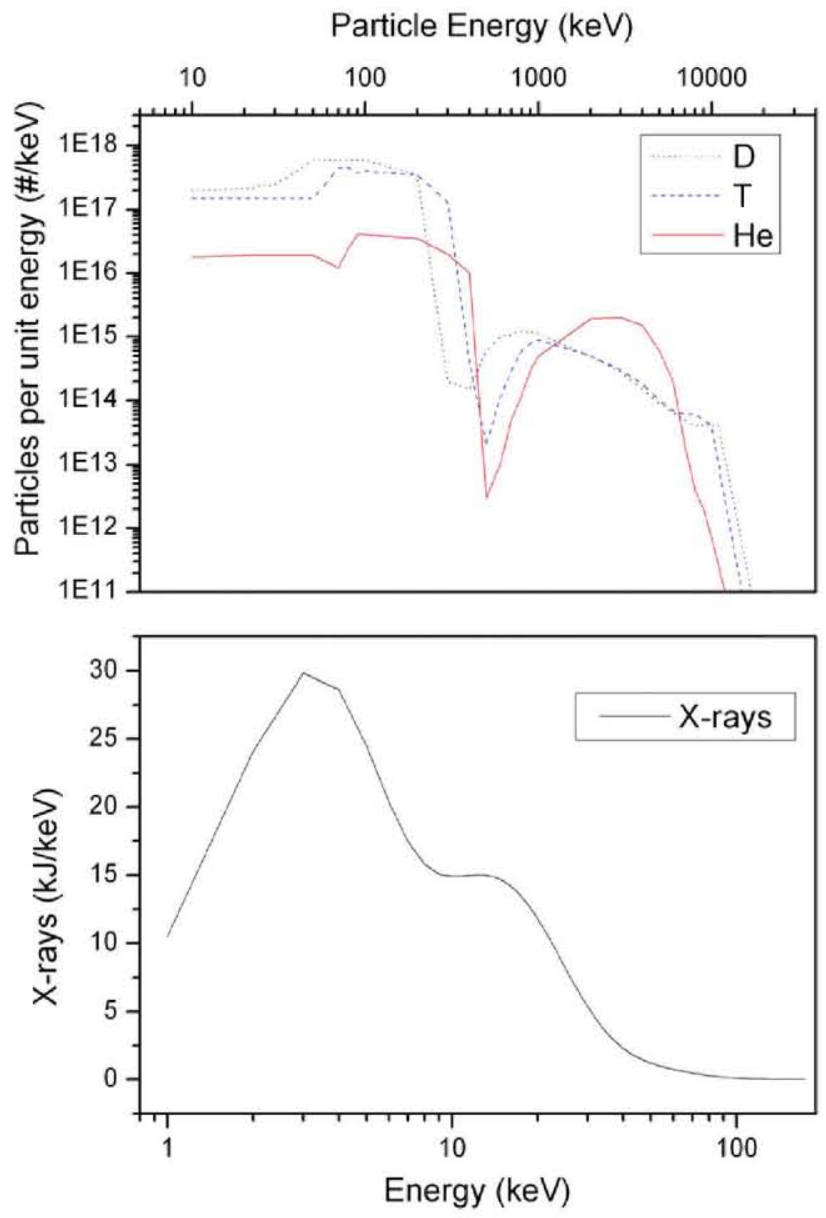

Figure 1. (Top) energy spectra of D,T and He, and (bottom) X-rays of a $48 \mathrm{MJ}$ shock ignition target.

simulation of the thermo-mechanical study of the armour. In the case of ions, this temporal profile can be calculated from their kinetic energy spectra of figure 1 . Considering a reaction chamber of $5 \mathrm{~m}$ in radius, the arrival time of $\mathrm{D}, \mathrm{T}$ and $\mathrm{He}$ particles is represented in figure 2. In the case of x-rays in which all photons travel at the same speed, the temporal profile comes determined by the time span in which they were created. According to previous calculations, the $\mathrm{x}$-ray pulse duration has been estimated to be around $1 \mathrm{~ns}$ [9].

Figure 2 shows how the different species contribute to the deposition of energy in time steps of $25 \mathrm{~ns}$ for a $5 \mathrm{~m}$ radius chamber. As can be seen, x-rays arrive almost immediately after the ignition, depositing their energy (6.3\% of the $10.7 \mathrm{MJ})$ at around $17 \mathrm{~ns}$. Then, the fast $\mathrm{D}, \mathrm{T}$ and $\mathrm{He}$ involved in the ignition arrive at around $150 \mathrm{~ns}$ after the explosion. Those fast particles have deposited their energy ( $50 \%$ of the $10.7 \mathrm{MJ}$ ) in the armour before the first $\mu$ s. Finally, slow D and T particles and thermal $\mathrm{He}$ arrive at the armour after the first $\mu$ s and deposit their energy (the remaining 44\%) during $1.5 \mu$ s. In around $2 \mu \mathrm{s}$, all the fusion energy that will be delivered to the armour, 10.7 MJ, is deposited.

Finally, the spatial deposition of that energy in the armour is also relevant for an accurate simulation of the thermomechanical behaviour of the armour. The spatial energy profile of the different ions can be calculated using the SRIM 


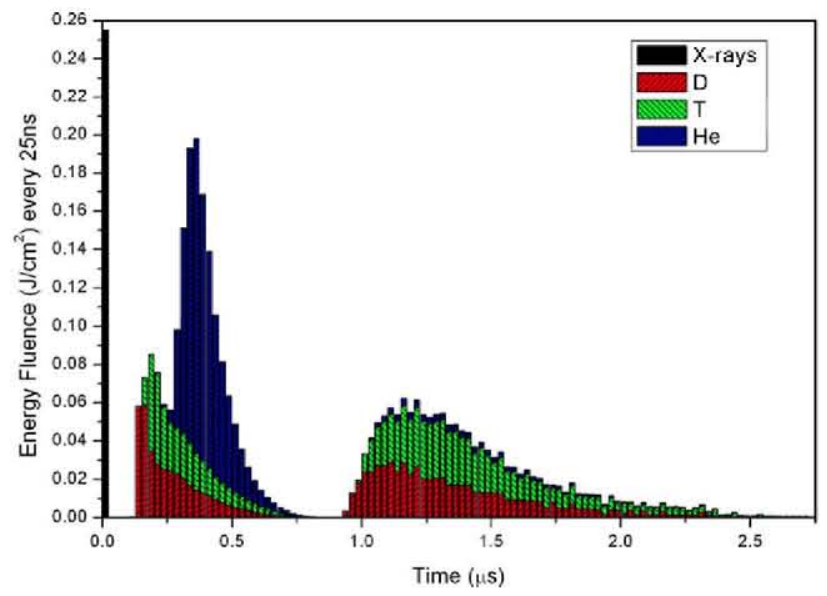

Figure 2. Temporal profile of the energy deposition of $\mathrm{x}$-rays, $\mathrm{He}, \mathrm{D}$ and $\mathrm{T}$ on a $5 \mathrm{~m}$ radius chamber for a $48 \mathrm{MJ}$ shock ignition target. Histograms correspond to time intervals of $25 \mathrm{~ns}$. Bars of the different species are added up to show the total energy deposition at each time step.

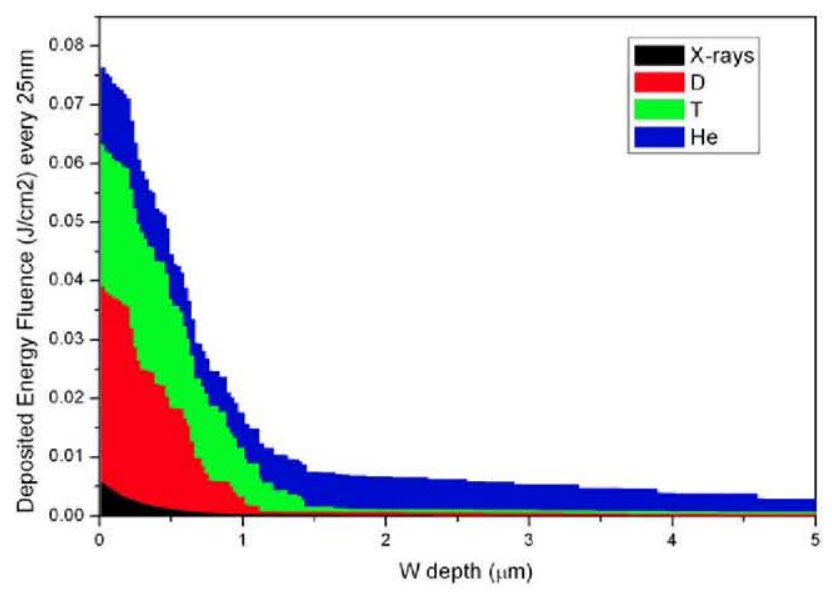

Figure 3. Spatial distribution of the deposited energy for the indicated particles.

$\operatorname{code}^{2}$. Considering tungsten as the armour material, SRIM simulations are presented in figure 3 . In the case of x-rays, the spatial deposition has been estimated using the absorption coefficient tables ${ }^{3}$ of x-rays on $\mathrm{W}$ in our spectral region of interest (figure 2). It is important to note that more than 50\% of the total energy is deposited just in the first micrometre. In the first two micrometres around $66 \%$ of the energy is deposited. This implies that all energy deposited at greater depths (as is the case of fast $\mathrm{D}$ and T ions, which penetrate some hundred micrometres, or energetic x-rays which travel some tens of micrometres) will play very little role in the thermomechanical behaviour of $\mathrm{W}$. A rough estimate indicates that around 15 to $20 \%$ of the arriving energy will be deposited in the $\mathrm{W}$ armour with almost no thermo-mechanical effect.

\section{Simulations and results}

In principle, HiPER has been proposed as a spherical chamber of $10 \mathrm{~m}$ in diameter with $1 \mathrm{~mm}$ tungsten armour as inner wall.

\footnotetext{
2 http://www.srim.org/

3 http://www.nist.gov/physlab/data/xraycoef/index.cfm
}

In order to simulate the thermal effects on the armour due to its exposure to radiation, we consider three different scenarios:

Scenario 1-Energy is deposited on the surface during some $\mu$ s continuously.

Scenario 2-Energy is deposited on the surface following the real temporal distribution.

Scenario 3-Energy is deposited taking into account the penetration depth of the different particles and the real temporal profile.

This exercise will show the importance of an accurate description of the spatial and temporal deposition of energy in the case of simulations for inertial fusion. The heat load on plasma facing materials takes place in such short pulses and with such broad energy spectra of species that approximations such as the ones used in magnetic confinement fusion (constant deposition of energy in the first micrometre of the material) are not valid. Thus, parameters such as the heat flux value [10] and the roughening and melting limits for tungsten should be handled with care.

The calculations have been carried out using the multiphysics open software CODE ASTER ${ }^{4}$. This software allows us to vary the physical and mechanical constants as a function of temperature which has been implemented for our calculations using the ITER material handbook ${ }^{5}$. In all cases and for simulation purposes, the $1 \mathrm{~mm} \mathrm{~W}$ armour is in contact with a fictitious coolant at a constant temperature of $600 \mathrm{~K}$. Also note that in these simulations, no other effect but the deposition of energy in the first wall is considered. Thus, atomistic effects such as sputtering, production of defects and changes in the chemical composition of the armour are not taken into account.

\subsection{Scenario 1 energy is deposited on the surface during some us continuously}

The simplest scenario possible in the deposition of energy on the armour is to consider that all the radiation energy is deposited evenly in time on the surface. Thus, for our case, the total amount of energy delivered by the x-rays, $\mathrm{D}, \mathrm{T}$ and $\mathrm{He}$ ions is $10.7 \mathrm{MJ}$, which corresponds to an energy fluence of $3.4 \mathrm{~J} \mathrm{~cm}^{-2}$ for a $5 \mathrm{~m}$ radius chamber. This energy can be considered as deposited in a time of $2 \mu$ s (see figure 2). This one-dimensional heat equation problem has an analytical solution and yields a maximum temperature value on the surface of $1900 \mathrm{~K}$. Taking into account the temperature dependence of the physical properties of $\mathrm{W}$, the simulation was performed using the CODE ASTER software. Figure 4(a) shows how temperature reaches a maximum of $2200 \mathrm{~K}$ at the end of the pulse. After $2 \mu \mathrm{s}$, the temperature decreases rapidly due to the high thermal conductivity of $\mathrm{W}\left(173 \mathrm{~W} \mathrm{~m}^{-1} \mathrm{~K}^{-1}\right)$, reaching the base temperature of $600 \mathrm{~K}$ after $10 \mathrm{~ms}$. Without further discussion, we can say that, since $\mathrm{W}$ can displace heat so quickly, the fact that the time profile of the deposition of energy is not considered will give us an underestimation of the maximum temperature.

\footnotetext{
4 http://www.code-aster.org/

5 Tungsten properties ITER Material Handbook http://aries.ucsd.edu/
} 

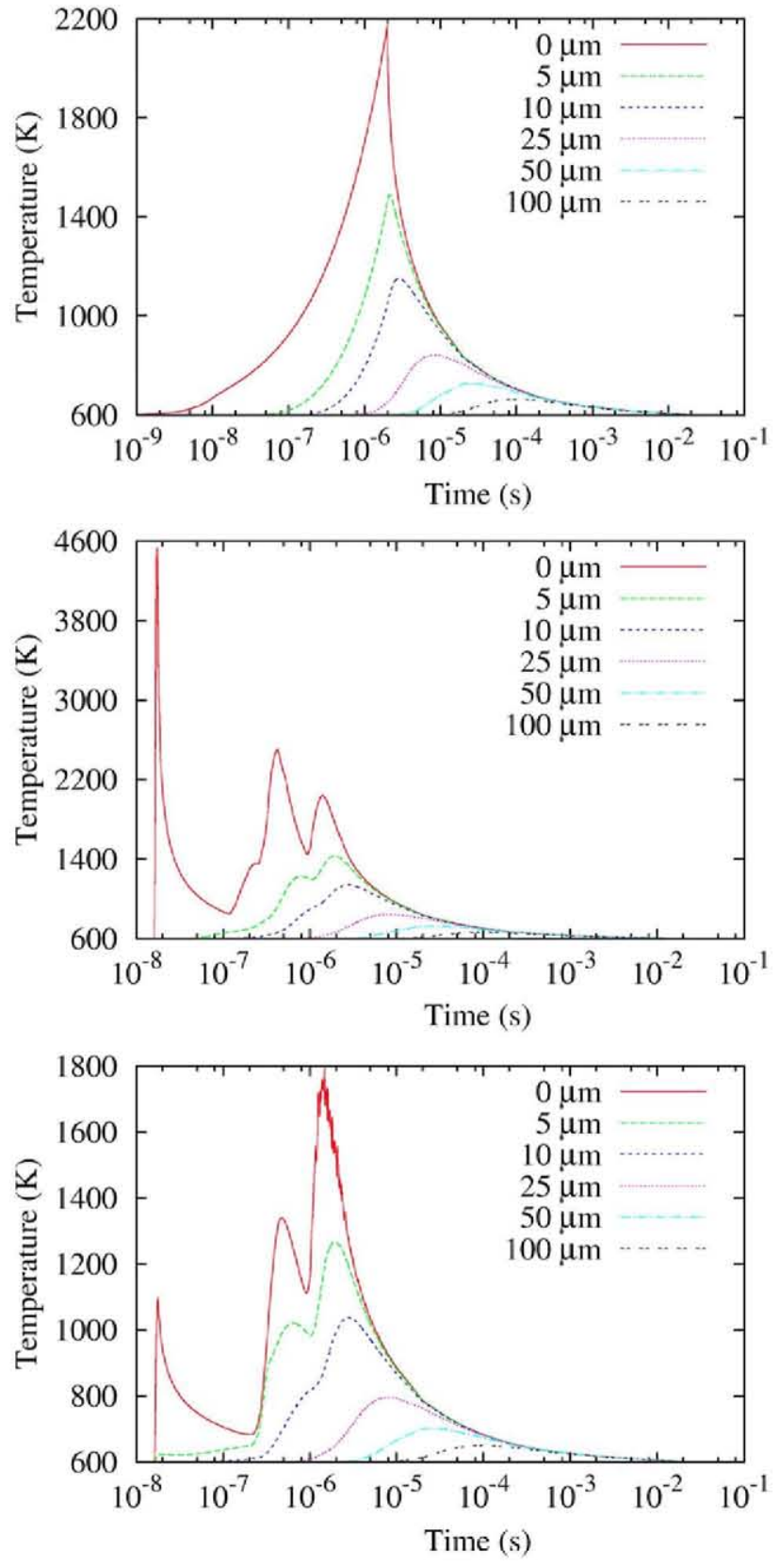

Figure 4. Temperature evolution at different tungsten depths for a heat load of $3.4 \mathrm{~J} \mathrm{~cm}^{-2}$ under (a) scenario 1 (top), (b) scenario 2 (middle) and (c) scenario 3 (bottom).

\subsection{Scenario 2-energy is deposited on the surface following the real temporal distribution}

The first refinement in our simulations to be considered is to include a proper temporal profile of the energy deposition on the $\mathrm{W}$ armour. As expected, simulations (figure $4(b)$ ) yield quite different results from that of scenario 1 . The first evident conclusion is that the temperature evolution of the armour follows the time structure of the radiation pulse, reaching at certain points higher temperatures and dropping to the base temperature after some ms. The most remarkable result is that, according to the simulations, the fast energy delivery of the $\mathrm{x}$-ray pulse leads to temperatures above the melting

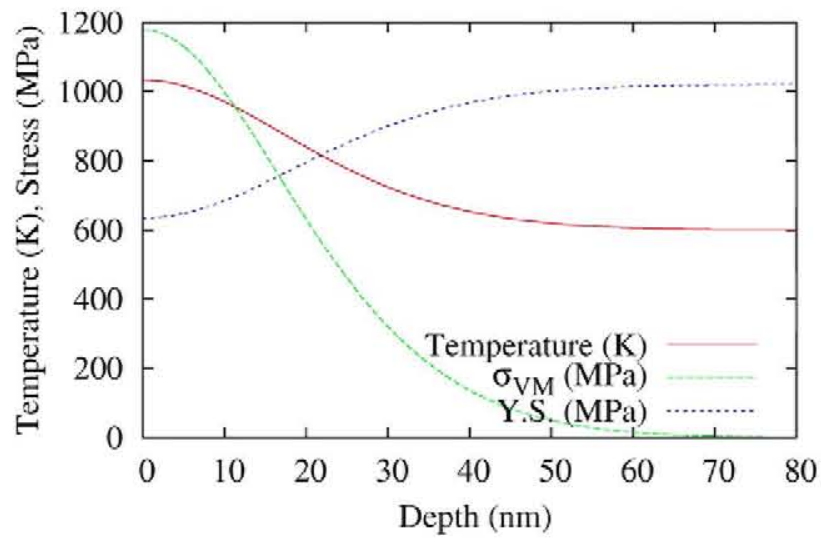

Figure 5. Temperature, Von Mises stress and yield strength as a function of depth at $1.5 \mu \mathrm{s}$, of simulation.

point $(3600 \mathrm{~K})$. However, experiments with $\mathrm{x}$-ray sources have shown that $\mathrm{W}$ starts to melt at much higher energy fluences [11]. This time, one must realize that the deposition of energy only on the surface obviates the fact that the radiation energy has a spatial profile inside the $\mathrm{W}$ according to the penetration depth of the different particles. This simplification in the modelling leads to an overestimation of the maximum temperatures.

\subsection{Scenario 3-energy is deposited taking into account the penetration depth of the different particles and the real temporal profile}

In this scenario, we consider the temporal and spatial profile of the energy deposition of the radiation in the $\mathrm{W}$ armour. A detailed description of the temporal and spatial distribution of energy differs from previous scenarios (figure $4(c)$ ). The temperature evolution at different depths clearly shows similar results to those obtained for other W armours [3] and the highest temperature does not reach $1800 \mathrm{~K}$. Once again, the $\mathrm{W}$ armour diffuses away all heat before the next pulse.

Under these proper conditions for the deposition of energy, we also carried out a $2 \mathrm{D}$ analysis on the mechanical behaviour of the W armour with CODE ASTER. The observed increase in temperature generates a measurable expansion of the $\mathrm{W}$ which, in turn, causes compressions in the wall. The main compression is tangent to the surface of the wall because $\mathrm{W}$ only can expand in the radial direction due to geometrical considerations. Figure 5 shows temperature and Von Mises stress $\left(\sigma_{\mathrm{VM}}\right)$, which measure the stress compression in the wall, as a function of the depth at a fixed time of $1.5 \mu \mathrm{s}$. When temperature increases, the expansion of the material produces an increase in the stress. The presented stress has been obtained considering the hypothesis that tungsten behaves as an elastic material, so when the Von Mises stress is higher than the yield strength (also represented in figure 5), the tungsten suffers plastic strain. The fact that at high temperature the yield strength decreases makes that crossing more favourable. Thus, plastic strain is located in the first micrometre of the wall, reaching around $18 \mu \mathrm{m}$ in times after $1.5 \mu \mathrm{s}$. When the temperature decreases and $\mathrm{W}$ returns to its initial volume, there is residual traction stress in this layer. As detailed by 
Blanchard and Martin [12], fatigue due to cycle plastic strain might produce cracks at the surface. The possibility that these cracks, together with other atomistic effects, influence the survivability of the $\mathrm{W}$ armour with increasing fusion shots is under debate [12].

\section{Conclusions}

The inner wall of an inertial fusion reaction chamber will have to withstand high power loads five to ten times a second. With no ion or $\mathrm{x}$-ray protection, only relative large chambers (5-8 $\mathrm{m}$ radius) and wall armour made of high resistant materials to thermal loads and mechanical stresses (such as tungsten) can handle this harsh environment. In order to appropriately study the thermo-mechanical effects of the fusion radiation on the armour, one has to fully account for the precise temporal and spatial deposition of the radiation on the material. Avoiding this will underestimate or overestimate the energy fluence limit $\left(\mathrm{J} \mathrm{cm}^{-2}\right)$ to preserve the lifetime of the armour. This point has been clearly demonstrated on these pages.

Moreover, this work shows the first thermo-mechanical studies on the $\mathrm{W}$ armour for the HiPER project under the radiation of a $50 \mathrm{MJ}$ shock ignition target. Calculations reveal that $\mathrm{W}$ armour will work well below its melting point. Only mechanical effects due to transitions of $W$ to its plasticity phase could affect its performance. Fatigue and eventually crack formation under hundreds/thousands fusion shots might end in mass loss and irreversible damage.

\section{Acknowledgments}

The authors want to thank the Spanish Ministry of Science and Innovation for its support under the program ACI-PROMOCIONA 2009.

\section{References}

[1] Raffray A.R., El-Guebaly L., Federici G., Haynes D., Najmabadi F., Petti D. and Aries-Ife team 2004 Fusion Sci. Technol. 46417

[2] Kaufmann M. and Neu R. 2007 Fusion Eng. Des. 82521

[3] Raffray A.R., Blanchard J., Latkowski J., Najmabadi F., Renk T., Sethian J., Sharafat S. and Snead L. 2006 Fusion Eng. Des. 811627

[4] Goto T., Someya Y., Ogawa Y., Hiwatari R., Asaoka Y., Okano K., Sunahara A. and Johzaki T. 2009 Nucl. Fusion 49075006

[5] Ueda Y., Kashiwagi H., Fukumoto M., Ohtsuka Y. and Yoshida N. 2009 Fusion Sci. Technol. 5685

[6] Moses E.I. 2008 Energ. Convers. Manag. 491795

[7] Zimmerman G., Kershaw D., Bailey D. and Harte J. 1978 J. Opt. Soc. Am. 68549

[8] Perkins J. 2009 private communication, Lawrence Livermore National Laboratory, USA

[9] Perkins J. 2005 private communication, Lawrence Livermore National Laboratory, USA http://www.docstoc.com/docs/ 54806737/Temperature-Response-and-Ion-Depositionin-the-1-mm

[10] Linke J., Escourbiac F., Mazul IV, Nygren R., Rodig M., Schlosser J. and Suzuki S. 2007 I. Nucl. Mater: 367-370 1422

[11] Tanaka T.J., Rochau G.A., Peterson R.R. and Olson C.L. 2005 J. Nucl. Mater. 347244

[12] Blanchard J.P. and Martin C.J. 2005 J. Nucl. Mater. 347192 